\title{
EAl Endorsed Transactions

\section{RapidNet IP: A synchronized, high throughput, low power wireless networking solution for ESL}

\author{
Kaveri Banakar, ${ }^{1, *}$ and Shruthi Shetty ${ }^{1}$ \\ ${ }^{1}$ Analog Devices, Bengaluru, India
}

\section{Abstract}

\begin{abstract}
Electronic shelf labels (ESL) are electronic equivalent of price tags in retail stores, product id labels in large warehouses, and instruction sheets in manufacturing assembly lines. The volume of labels varies up to 1000-12000 in numbers depending on the application. This paper describes novel wireless protocol RapidNet IP and data compression algorithm used to update the ESL tags remotely. RapidNet IP is a secure \& synchronized high throughput, low power \& long-range wireless networking protocol. This can update 12000 ESL tags in one hour. Time Synchronized channel hopping (TSCH) method allows for congestion free traffic for larger number of nodes. The proprietary joining method allows the faster network formation. Data compression algorithm developed as a part this protocol compresses ESL tag PNG image from $150 \mathrm{~kb}$ to $2 \mathrm{~kb}$. This facilitates the ability to update more tags in a short period and reduces the nodes wakeup time. This enables the low power wireless network end nodes battery life up to 7 years (with CR2450 x2 (2.9” ESL tag)).
\end{abstract}

Received on 21 April 2019; accepted on 30 May 2019; published on 16 July 2019

Keywords: IoT, ESL, low power wireless protocol for large network.

Copyright (C) 2019 Kaveri Banakar et al., licensed to EAI. This is an open access article distributed under the terms of the Creative Commons Attribution license (http://creativecommons.org/licenses/by/3.0/), which permits unlimited use, distribution and reproduction in any medium so long as the original work is properly cited.

doi:10.4108/eai.16-7-2019.162213

Corresponding author. Email: Kaveri.Banakar@analog.com

\section{Introduction}

ADI RapidNet IP protocol is a sub-GHz synchronized wireless networking solution based on low power wireless networking standards [1], that address industrial and commercial applications where reliability, robustness, scalability and battery life are critical. This is differentiated by high data throughput for large networks, especially in the downlink direction where it can update thousands of end nodes with kilobytes of data in less than an hour. The protocol also facilitates fast response time allowing end nodes to send important alert messages with only several minutes latency in a very large network.

The motivation to develop protocol being develop a network capable of updating 12000 nodes per hour with communication robustness .The network should support low power mode to ensure 7-year battery life with cr2450 $\mathrm{x} 2$. This network allows ESL application to perform dynamic update of the EINK displays from the PNG image sent from the gateway. Dynamic update enables the retailers to fluctuate pricing to match demand, online competition, inventory levels, shelf-life of items, and to create promotions.

The main challenge for this protocol being considered is congestion control in a very large network and time synchronized communication between nodes and gateway. Time synchronized channel hopping is being implemented as part of the protocol to address this issue. The currently available solution in the market with IR communications requires continuously powering of the nodes which will terminates 7-year battery life requirement. The other low power wireless protocol solutions are not capable of handling larger networks efficiently. Hence there was a requirement to develop a high throughput, low Power and long-range wireless networking solution.

The RapidNet IP wireless network consists of 3 network components: gateway, access point and end node. End nodes will be interface with ESL display device. The PNG image to be displayed on the ESL tag is sent from the gateway. User chooses the application specific PNG image from the web or stored labels. The image data for these labels varies significantly from application to 
application and cannot be generated on the node using metadata sent over the air, hence the whole image needs to be created on the gateway/cloud and transferred to these nodes. Since the RAW image data could be up to $150 \mathrm{kB}$ for a 5.6" display it requires compression so that it can be transferred to the nodes using low power wireless networks. This chosen labels from the web for update on the node must go through specific generalized compression algorithm to make ESL compatible to support all types of image of any size. This compression algorithm makes the embedded application simpler, optimizes memory requirements and to expand it to variety of use cases. Currently ESL application supports 2 types of display 2.9" and 5.65" displays, this application can be expandable to variety larger displays to support different use cases.

\section{RapidNet IP}

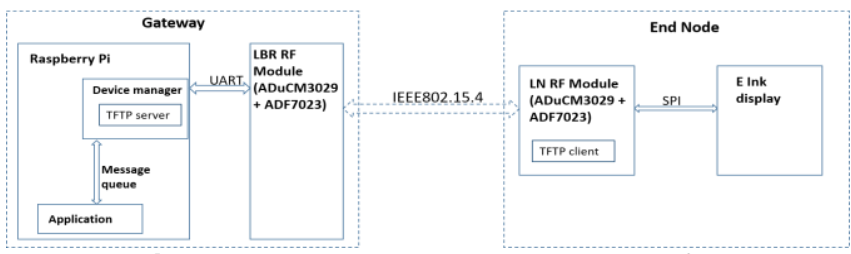

Figure 1. RapidNet IP components figure

RapidNet IP is a complete point-to-multipoint wireless networking solution operating in the sub-GHz frequency range with regional support for the major worldwide markets. The wireless protocol uses a time synchronized, star-repeater network topology. enabling networks of thousands of nodes to communicate with higher data throughput, more power efficiently and extending battery life. The RapidNet IP wireless network protocol runs on either the ADuCM3029 or ADuCM4050 ultra low power ARM cortex M4-based microcontroller and ADF7023 low power radio transceiver. The network components are listed below:

ESL-Gateway(GW): ADuCM3029 microcontroller with ADF7023 transceiver is connected to the gateway with USB interface.

ESL-Access point: ADuCM3029 microcontroller with ADF7023 transceiver.

ESL-End node: ADuCM3029 microcontroller with ADF7023 transceiver used as the end node which is integrated to the electronic display.

\subsection{Time Synchronized channel hopping (TSCH)}

TSCH is time synchronized communication and channel hopping to provide network robustness. Time slotted communication links increase potential throughput by minimizing unwanted collisions. Channel hopping extends the effective range of communications by mitigating the effects of multipath fading and interference. All devices share common time and channel information, devices may hop over the entire channel in a slotted way to avoid collisions, minimizing the need for retransmissions.

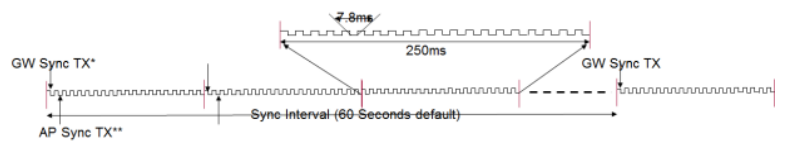

Figure 2. Time slot in RapidNet IP

In this protocol, time is sliced into timeslots. Each slot is $7.8125 \mathrm{~ms}$ and timeslots are grouped to form one slotframe. Slotframe repeats over time. One slotframe consists of 32 slots which will make one channel block(250ms). Channel hopping occurs every $250 \mathrm{~ms}$. Every slot interval (currently $60 \mathrm{sec}$ ), GW / AP will send the sync message. The sync message between them is 16 slots $(125 \mathrm{~ms})$ apart. Multiple AP's will send Sync messages on different channel offsets. Nodes will sync to the network on reception of sync message. Using this message, nodes will adjust their timing and get the information for the next communication. Sync message also has the operating mode for next sync interval.

\subsection{Protocol definition}

Network discovery happens after receiving the sync message. The network discovery is based on the RPL messages (DIO message) [2]. The nodes will join to the GW based on the RPL DIO message information. The GW can upload image file to the nodes using TFTP protocol. TFTP runs on UDP sockets.

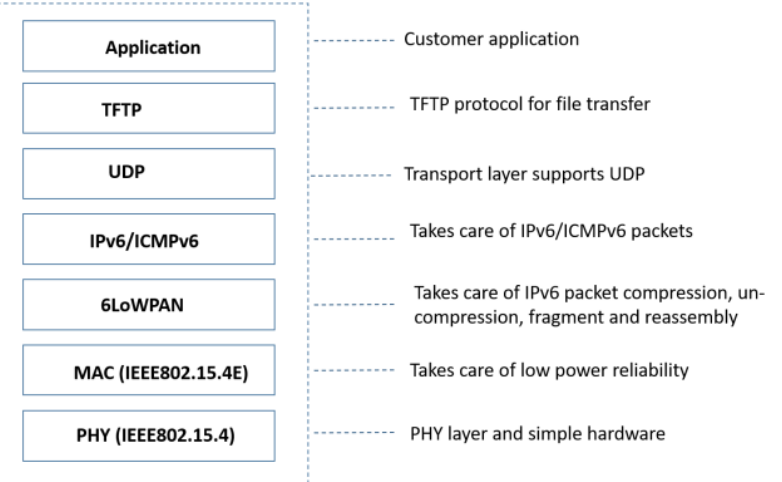

Figure 3. RapidNet IP stack used by ESL network components

The different phases of RapiNet IP network are:

\section{Join phase}

The network starts by broadcasting sync packets from LBR to all the nodes in the network. The time between 2 sync packets is called as sync interval. The sync Interval is user configurable. The sync packet contains the time information of the LBR, absolute slot number (ASN), other proprietary information necessary to join the network. Once node receives the sync message, it syncs its time with LBR, then it sends registration request in 
appropriate slot based on the information received in the sync packet to the gateway. The gateway will send the response if it is an authorized node. Upon receiving registration response, node will be part of the network and goes to low power mode. It will be in low power mode until the next sync interval. The nodes which are not in the range of the gateway will receive the sync packet from access points. The access points forward the sync message sent from gateway in different channels. This avoids the contention and enables the large nodes joining within few minutes.

\section{Operating phase}

When a new data arrives at gateway, it waits for next sync packet and sends a download notification to all the nodes. Download notification field contains the device ID of the nodes and number of slots allotted to complete the data download. Node sends read request to the gateway to receive the file in its allocated slot. Once the allocated slot time expires the node enters low power mode. TFTP protocol [3] is used for reliable image file transfer from the gateway to the node. The gateway compresses the image before sending to the node. If the compressed image is more than $1 \mathrm{~KB}$, it creates the TFTP blocks with size $1 \mathrm{~KB}$. The gateway keeps record of missed TFTP blocks based on the received ACK packet. It reschedules the slots to send missed TFTP blocks in next sync interval. The gateway can schedule 1 TFTP block of size $1 \mathrm{~KB}$, per node for 250 nodes in 60 seconds.

\section{OTA phase}

In very large and dense network with thousands of nodes, it is difficult to update individual nodes. In over-the-air operating mode, the new firmware image will be broadcasted by the gateway to all the nodes. All the nodes will be in receive mode until it receives all the firmware blocks. Missed blocks will be requested by the end nodes and gateway will send these blocks as unicast packets to the respective nodes.

Storage phase

This is a special mode used in the warehouses to keep the devices very low power mode before deployment. This is not a normal mode, here a special beaconing device is used to configure all devices in the store. It consumes 23ua while compared to $9-10 \mathrm{uA}$ while it is in the normal operating low power mode.

\section{PNG compression and decompression algorithm \\ PNG Compression algorithm at the gateway uses PNG encoding tool.}

Two steps were implemented in the PNG encoding algorithm

(i) Palette indexed color method

This This technique provides the image compression up to $2 \mathrm{~kb}$.
In this technique, first PNG file is decompressed to RGB values which has multiple color values. Then RGB values are compressed as 3 color information, since only 3 colors are supported in the displays supported by the ESL. The three colors information stored in a separate piece of data is called a palette.

These palettes correspond to the indexes of each color and thus provides prominent level of compression by storing the color information in the form of indexes.

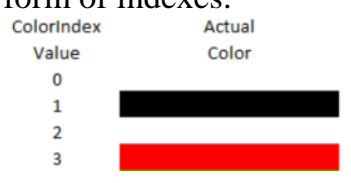

Figure 4. Palette indexes of colors

(ii) Sliding window of LZ77 compression optimization By default, the PNG compression sliding window is of 32768 bytes. But end node is a memory constrained device, it does not support $32 \mathrm{~KB}$ of sliding window. Hence one level of optimization is provided at the gateway by reducing the sliding window to 1024 bytes.

PNG Decompression algorithm at the node uses PNG decoding method

Two steps were implemented in the PNG decoding algorithm

(i) Line by Line decoding technique

This approach works by reorientation of the PNG image, thus achieved to reduce the RAM memory from $150 \mathrm{~kb}, 10 \mathrm{~kb}$. Decompresses only one line of PNG file at once, this corresponds to the width bytes of the PNG file. Send one line of decoded pixels to the display without storing it in the frame buffer. This process repeats for height number of times. This eliminates the necessity of frame buffer to store raw data in case of regular PNG technique.

(ii) Sliding window of LZ77 decompression optimization

Sliding window set to 1024 . This is possible because we are compressing the data at the gateway using the 1024 bytes sliding window and the same can be decoded at the nodes.

\section{Experimental results}

Network formation and image download period are tabulated for different network size.

Table 1. Network formation and image download period for network size

\begin{tabular}{lll}
\hline Network size & $\begin{array}{l}\text { Network formation } \\
\text { time in seconds }\end{array}$ & $\begin{array}{l}\text { Image download period } \\
\text { for 1KB in seconds }\end{array}$ \\
\hline 16 nodes & 4 & 4.5 \\
32 nodes & 8.5 & 9
\end{tabular}




\begin{tabular}{lll}
\hline 64 nodes & 16.5 & 17 \\
128 nodes & 33 & 33.5 \\
256 nodes & 64 & 64.5 \\
\hline
\end{tabular}

\section{Acknowledgements.}

We would like to thank Prakash Govindaraju, Reginald Dsouza, Balaji Dorairaj, Suresh Haralayya, Pranit Jadhav and Sharath Rao.

\section{References}

[1] IEEE 802.15.4 standard.

[2] RFC 6550: RPL: IPv6 Routing Protocol for Low-Power and Lossy Networks.

[3] RFC 1350: Trivial File Transfer Protocol (Revision 2). 